\title{
Las Aventuras Químicas de Sherlock Holmes: un recurso "elemental” en el aula de ciencias
}

Jorge Martín-García - Universidad de Zaragoza

$0000-0002-2247-0826$

Recepción: 27.12.2021 | Aceptado: 03.01.2022

Correspondencia a través de ORCID: Jorge Martín-García

iD 0000-0002-2247-0826

Citar: Martín-García, J (2022). Las Aventuras Químicas de Sherlock Holmes: un recurso "elemental” en el aula de ciencias. REIDOCREA, 11(3), 28-43.

Área o categoría del conocimiento: Didáctica de las Ciencias Experimentales

Resumen: En estos años de globalización, de realidades en incesante movimiento y continuos cambios la educación científica se ha convertido en una necesidad básica para cualquier individuo. En este contexto, la didáctica de las ciencias reclama una renovación profunda que permita recuperar el dinamismo inherente a esta disciplina; una pedagogía renovada que promueva aprendizajes verdaderamente significativos y provechosos con los que caminar hacia una verdadera alfabetización científica. Este artículo supone la continuación de trabajos previos centrados en el empleo de la narrativa como recurso didáctico en Física y Química. En él se proporcionan una serie de actividades y propuestas de aprendizaje situado con las que abordar diferentes contenidos de química en educación secundaria desde una perspectiva más amena y motivadora.

Palabra clave: Recursos didácticos

The Chemical Adventures of Sherlock Holmes: An "Elementary" Resource in the Science Classroom

Abstract: We live in a globalized world where everything changes at great speed, unceasingly. In these days scientific education has become a basic need for each and every individual. In this context, science teaching must be renewed in order to regain its inherit dynamism and to generate truly significant and useful leanings that allowed our society to reach a real scientific literacy. This article is the second part of a series of proposals including the use of narratives as a teaching aid in Physics and Chemistry. In this case, several context-based activities that can be used in high school chemistry subjects which may be used to increase students' motivation and their appreciation of science are presented.

Keyword: Teaching aids

\section{Introducción}

En trabajos anteriores (Martín y Vicente 2019) se comenzó a desarrollar y dar forma a una propuesta de trabajo basada en el empleo de la narrativa, los relatos e historias, como recursos didácticos en la enseñanza de las ciencias en educación secundaria. En este artículo, se presentan una serie de actividades con las que continuar dicha línea de trabajo.

El amor por la Química del personaje por antonomasia de la novela detectivesca, Sherlock Holmes, no ha pasado desapercibido para sus fans, a quienes ha servido de influencia e inspiración. Probablemente el ejemplo más claro de este hecho lo podamos encontrar en la serie de relatos recogidos bajo el nombre "The Chemical Adventures of Sherlock Holmes", publicados durante más de 15 años en el Journal of Chemical Education por los profesores Thomas G. Waddell y Thomas R. Rybolt, de la Universidad de Tennessee. En ellos se presentan una serie de casos, perfectamente contextualizados en la Inglaterra victoriana, en los que Holmes, acompañado de su inseparable amigo y biógrafo, el Doctor Watson, hace gala de unos insuperables conocimientos de Química, que pone al servicio de la ley para dar solución a una serie de misterios de lo más intrincados: 
Tabla 1: "Las Aventuras Químicas de Sherlock Holmes", relatos originales

\begin{tabular}{|c|c|c|}
\hline Título & Relación con la Química & Referencia \\
\hline $\begin{array}{l}\text { Sherlock Holmes and the } \\
\text { Yellow Prisms }\end{array}$ & Análisis químico cualitativo orgánico e inorgánico. & (Waddell \& Rybolt, 1989) \\
\hline $\begin{array}{l}\text { Sherlock Holmes and the } \\
\text { Fraudulent Ketone }\end{array}$ & $\begin{array}{l}\text { Método científico y procedimiento de trabajo en el } \\
\text { laboratorio. }\end{array}$ & (Waddell \& Rybolt, 1990) \\
\hline A Christmas Story & Análisis químico cualitativo. & (Waddell \& Rybolt, 1991) \\
\hline $\begin{array}{l}\text { The Case of the Screaming } \\
\text { Stepfather }\end{array}$ & Análisis químico cualitativo. & (Waddell \& Rybolt, 1992) \\
\hline $\begin{array}{l}\text { The Case of the Stoichiometric } \\
\text { Solution }\end{array}$ & Cálculos estequiométricos. & (Waddell \& Rybolt, 1993) \\
\hline The Hound of Henry Armitage & $\begin{array}{l}\text { Propiedades físicas y ajuste de reacciones } \\
\text { químicas. }\end{array}$ & (Waddell \& Rybolt, 1994) \\
\hline $\begin{array}{l}\text { The Problem of Woolthshrap } \\
\text { Prison }\end{array}$ & $\begin{array}{l}\text { Observación científica y uso criminal de } \\
\text { reacciones químicas. }\end{array}$ & (Rybolt \& Waddell, 1995) \\
\hline $\begin{array}{l}\text { Sherlock Holmes and the } \\
\text { Nebulous Nitro }\end{array}$ & $\begin{array}{l}\text { Observación científica, capacidad de } \\
\text { razonamiento y química orgánica. }\end{array}$ & (Waddell \& Rybolt, 1996) \\
\hline The Baker Street Burning & $\begin{array}{l}\text { Química forense, propiedades físicas y análisis } \\
\text { cualitativo orgánico. }\end{array}$ & (Waddell \& Rybolt, 1998) \\
\hline $\begin{array}{l}\text { The Death Puzzle at 221B } \\
\text { Baker Street }\end{array}$ & Química médica y análisis cualitativo. & (Rybolt \& Waddell, 1999) \\
\hline The Ghost of Gordon Square & Química de la magia. & (Waddell \& Rybolt, 2000) \\
\hline The Shroud of Spartacus & $\begin{array}{l}\text { Propiedades de las sustancia biológicas, análisis } \\
\text { cualitativo y test de detección de sangre. }\end{array}$ & (Waddell \& Rybolt, 2001) \\
\hline The Case of Three & $\begin{array}{l}\text { Propiedades físicas y caracterización química de } \\
\text { metales. }\end{array}$ & (Rybolt \& Waddell, 2002) \\
\hline The Blackwater Escape & $\begin{array}{l}\text { Análisis cualitativo inorgánico, observaciones en } \\
\text { el laboratorio y procesos redox }\end{array}$ & (Waddell \& Rybolt, 2003) \\
\hline Autopsy in Blue & $\begin{array}{l}\text { Análisis cualitativo inorgánico, Química forense y } \\
\text { sustancias con propiedades medicinales }\end{array}$ & (Waddell \& Rybolt, 2004) \\
\hline
\end{tabular}

Cada historia se centra en un tema distinto, con unos contenidos diferentes. Sin embargo, todas tienen la misma estructura. Existe una primera parte en la que se presenta el crimen y se describen, detalladamente, todos los experimentos y análisis que realiza el protagonista. En la segunda parte se recoge la solución del misterio, explicando las reacciones que se han producido o los cálculos que se deben llevar a cabo. De esta forma, la idea es suministrar la primera parte al estudiante y que sea él quien intente resolver el enigma.

El éxito de estas historietas ha sido rotundo, los autores reportan que desde que comenzaron con la serie, no han dejado de recibir cartas de otros profesores agradeciéndoles su trabajo, pidiéndoles permiso para emplear los relatos en clase y rogándoles que amplíen la colección con algún nuevo caso. Por ello, no es de extrañar que, años más tarde, Ken W. Shaw, un profesor americano de secundaria, aportara tres nuevos casos a tan impresionante archivo:

Tabla 2: Ampliación de "Las Aventuras Químicas de Sherlock Holmes"

\begin{tabular}{lll}
\hline Título & Relación con la Química & Referencia \\
\hline The Serpentine Remains & Análisis cualitativo, Química descriptiva y Química forense. & (Shaw, 2008) \\
Mrs. Hudson's Golden Brooch & Análisis cualitativo, metalurgia y análisis gravimétrico. & (Shaw, 2009) \\
The Brief Case of the Vile Humour & Análisis cualitativo & (Shaw, 2012) \\
\hline
\end{tabular}

Los conceptos involucrados en los relatos en general exceden los exigidos en el sistema educativo español. No obstante, con un pequeño trabajo previo de adaptación pueden convertirse en un recurso de gran interés con el que mostrar cuanto ha evolucionado la ciencia de la química y acercar a situaciones relativamente cotidianas los conocimientos adquiridos en el aula. 


\section{Propuesta de trabajo}

\section{The Case of the Screaming Stepfather:}

La historia seleccionada, (Waddell y Rybolt, 1992), ha sido previamente empleada como recurso didáctico (Montagut, Sansón, y González, 2005), para trabajar en asignaturas de Química General con alumnos universitarios. Sin embargo, el planteamiento que se desarrollará a continuación se dirige a otro tipo de alumnado, con otras características y por tanto con unos objetivos muy distintos. Se ha elegido este relato porque aporta una visión práctica, aplicada, de algunos de los conocimientos que se han de adquirir en la etapa de secundaria, lo que lo convierte en un elemento altamente motivante con el que profundizar en los conocimientos de los estudiantes.

\section{Resumen del relato:}

Un joven muchacho acude una noche al 221B de Baker Street en busca de Sherlock Holmes, pues su padrastro Mr. Wooly, se encuentra gravemente enfermo. Holmes y Watson acuden a la casa del joven, donde descubren un frasco de grosellas de un impresionante color verde. Tras unos oportunos análisis químicos, en los que una cuchara de acero parece convertirse en una de bronce, el detective concluye que $\mathrm{Mr}$ Wooly ha sido envenenado, descubriendo, al mismo tiempo, el veneno y al envenenador.

$<$ Holmes smiled and turned to me. "Our poisoning is solved, Watson. Our poisoner is identified. >>

(Waddell y Rybolt, 1992)

\section{Propuesta de trabajo:}

Se propone es entregar a los estudiantes las dos partes del relato, la narración y la solución, y hacer un análisis de los mismos, a partir del cual puedan plantearse una serie de cuestiones y prácticas de laboratorio:

1. Cuestiones sobre el texto:

a. Sobre la reacción que se produce en la cuchara de Holmes, responde:

i. Escríbela e indica que especie es la oxidante y cuál la reductora.

ii. Presenta las dos semirreacciones que se producen con sus potenciales estándar de reducción

iii. Justifica que la reacción es espontánea.

b. Explica qué es una reacción de precipitación y comenta alguna aplicación analítica de este tipo de reacciones.

c. El acetato de cobre es una sal, que pueda actuar como una base orgánica de Arrhenius, y que está constituida por dos grupos acetato $\left(\mathrm{CH}_{3}-\mathrm{COO}{ }^{(-)}\right)$ y un catión $\mathrm{Cu}^{2+}$.

i. ¿Qué crees que se produce cuando se hace reaccionar con ácido sulfúrico?

ii. ¿Qué productos se forman? Nómbralos. 
d. Predice y da nombre a los productos generados en la reacción entre el ácido acético y el alcohol de grano (etanol).

e. ¿Qué tipo de reacción orgánica es? Comenta otros tipos de reacciones orgánicas que conozcas, explicando en qué consisten y aportando un esquema general o un ejemplo de las mismas.

2. Obtención de cobre por cementación:

$\ll$ Holmes grabbed the closest jar and forced it open. He inserted his steel spoon and began to crush and stir the contents of the jar with his steel spoon. To my amazement when the steel spoon was removed from the jar, it had been transformed, as if by the magic of alchemy, to a shiny bronze-like metal [...]

A layer of copper metal formed on the spoon and this was the cause of the seeming transformation. The steel spoon established the presence of copper in the store's own gooseberries. The cupric ions plate out, reduced by the iron in the spoon. >>

(Waddell y Rybolt, 1992)

El fragmento anterior, combinado con algunas de las cuestiones planteadas en el ejercicio anterior, ofrecen el fundamento teórico de una de las prácticas más clásicas de química inorgánica, la obtención de cobre por cementación. De esta forma, la lectura permite plantear una experiencia de laboratorio con la que trabajar contenidos procedimentales, desarrollar las habilidades de laboratorio del estudiantado y repasar contenidos como reacciones redox, cinética, catálisis...etc.

Para ello se elaborará y entregará un guion (ver Anexo I) al alumnado, en el que se indique, al menos, el objetivo que se persigue, el procedimiento experimental que ha de seguirse para el desarrollo de la experiencia y las cuestiones complementarias que se consideren pertinentes; así como las instrucciones precisas para la elaboración de un informe de laboratorio con el que se concluirá la experiencia.

\section{Reacciones de formación de complejos:}

$<<$ I had my suspicions, Watson, and in confirmation, I took some of the gooseberry juice, diluted it to mask the green color, and added ammonia solution to it. A deep blue color resulted. Mark that, Watson, it is critical Moreover, acidification with hydrochloric acid caused the blue solution to become nearly colorless again. Treatment then with potassium ferrocyanide, $\mathrm{K}_{4} \mathrm{Fe}(\mathrm{CN})_{6}$, gave a distinct red precipitate. I have been working all night at my lab bench. >>

(Waddell y Rybolt, 1992)

Este fragmento del relato, además de repasar los conceptos asociados a las reacciones de precipitación permite ampliar los contenidos de $2^{\circ}$ de Bachillerato para incluir el estudio cualitativo de las reacciones de formación de complejos. Por otro lado, con esta actividad se pretende que los estudiantes se familiaricen con el manejo del material y la forma de trabajar de los químicos, y que, al mismo tiempo, disfruten de una experiencia interesante y motivadora, en la que se llevarán a cabo reacciones de química recreativa, bastante llamativas.

Se propone, en primer lugar, la observación de la reacción entre el catión Fe3+, y el anión tiocianato, $\mathrm{SCN}^{(-)}$, para formar el catión complejo tiocianato de hierro (II), $[\mathrm{FeSCN}]^{2+}$, partiendo del procedimiento estipulado por Pérez y Gil (2012), quienes la 
emplean para reproducir una escena de película de 1950 "Los diez mandamientos" y de acuerdo con el guion que puede encontrarse en el Anexo II.

En la segunda parte de la experiencia, se insiste en la observación de este tipo de reacciones, en particular la formación de complejos amoniacales de cobre, caracterizada porque dota a la disolución de un color azul relativamente intenso, y que ha sido, y puede ser, empleada para la detección de amoníaco en aguas residuales, de forma incluso cuantitativa, por colorimetría. Con esta actividad se refuerzan, nuevamente, las conexiones entre la ciencia que se enseña en la escuela y los problemas reales de la sociedad en que vivimos, mostrando las aplicaciones prácticas de aquello que hemos de estudiar.

\section{The Case of the Stoichiometric Solution.}

Con esta, la quinta entrega de la serie (Waddell y Rybolt, 1993), se pretende ofrecer al alumnado una visión diferente, más amena e interesante, más conectada con su vida cotidiana y, en definitiva, más motivante, de algunos de los contenidos que constituyen el programa de la asignatura de Física y Química.

\section{Resumen del relato:}

Un extraño asesinato ha tenido lugar en la sala de explosivos de una cantera de piedra caliza. La víctima no presenta signos de ningún tipo de violencia, y ha sido encontrada en una habitación cerrada, que solo puede ser candada desde fuera y que consta exclusivamente de un pequeño orificio para la ventilación. Sherlock Holmes se hará cargo del caso y tras una inspección del escenario del crimen y el interrogatorio del vigilante de la cantera, se encontrará en disposición de desentrañar el misterio:

$\ll$ "Stoichiometry, Watson, stoichiometry," he mumbled when I inquired as to what he was doing. Soon he snatched a second notebook page from Gregson, wrote out a sentence or two, and handed the page back to the Inspector. >>

(Waddell y Rybolt, 1993)

\section{Propuesta de trabajo:}

Se propone la división del relato original en tres textos (ver Anexo III), que se irán entregando paulatinamente a los alumnos a medida que se vaya avanzando en el desarrollo de la actividad. Los dos primeros fragmentos se destinan al repaso y refuerzo de contenidos, para lo que incorporan una serie de cuestiones que cada estudiante deberá responder individualmente:

\section{Actividades del Texto 1:}

< The addition of acetic acid to calcium carbonate yields carbon dioxide gas. >>

(Waddell y Rybolt, 1993).

a. La reacción a la que se refiere Holmes al final del texto es la siguiente ${ }^{1}$ :

$$
\mathrm{CaCO}_{3}(\mathrm{~s})+\mathrm{CH}_{3} \mathrm{COOH}(\mathrm{l}) \rightarrow \mathrm{Ca}\left(\mathrm{CH}_{3} \mathrm{COO}\right)_{2}(\mathrm{l})+\mathrm{CO}_{2}(\mathrm{~g})+\mathrm{H}_{2} \mathrm{O}
$$

\footnotetext{
${ }^{1}$ En cursos superiores, donde ya se haya impartido formulación orgánica, se recomienda que no se facilite la reacción a los estudiantes, y que sean ellos los que la escriban a partir del fragmento del texto, a fin de trabajar un poco los contenidos sobre formulación.
} 
i. Ajústala y explica de qué tipo de reacción se trata.

ii. Como puedes ver en ella aparecen sustancias tanto sólidas como líquidas y gaseosas.

1. ¿Cuáles son las principales propiedades de cada uno de estos estados de agregación? Explícalos de acuerdo a la teoría cinético molecular de los gases.

2. Realiza un esquema en el que se describan los procesos por los cuales una sustancia puede cambiar de estado, indicando el estado en que se encuentra tanto antes como después del proceso.

iii. Considerando la reacción ¿Se te ocurre cuál fue la causa de la muerte de Mr. Bishop?

b. Teniendo en cuenta que $1 p^{3} e^{3}=0,0283 \mathrm{~m}^{3}$ y que Holmes ha estimado que el tamaño de la habitación es de 7 pies de ancho, 7 pies de largo y 8 pies de alto, calcula el volumen de la estancia.

i. ¿Cuántos moles de aire, en condiciones normales, habría en la habitación?

ii. ¿Cuántos moles serían de oxígeno y cuántos de nitrógeno?

iii. ¿Cuál sería la presión total existente en la habitación?

iv. ¿Cuáles serían las presiones parciales de cada uno de los dos componentes anteriores?

\section{Actividades del Texto 2}

a. Sabiendo, tal como nos recuerda Sherlock, que el vinagre contiene un $5 \%$ de ácido acético $\left(\mathrm{CH}_{3} \mathrm{COOH}\right)$ y que su densidad es de $1050 \mathrm{~g} / \mathrm{l}$, determina:

i. El volumen y la masa de vinagre que necesitamos para llenar de $\mathrm{CO}_{2}$ todo el volumen de la habitación en condiciones normales.

b. El ácido acético glacial $\left(\mathrm{CH}_{3} \mathrm{COOH}\right)$, se vende en botellas de $1 \mathrm{~L}$ y que tiene aproximadamente la misma densidad que el vinagre, calcula:

i. La masa de ácido acético glacial necesario para producir un volumen de $\mathrm{CO}_{2}$ al volumen de la habitación.

ii. La cantidad de botellas de ácido que necesitarías.

c. Reflexiona sobre la siguiente frase de Holmes:

$<$ "I may not otherwise have noticed the sour-smelling hole and the deep wheel ruts, which are very much related to the case." >

(Waddell and Rybolt, 1993)

d. ¿Sabrías decir ya quien cometió el asesinato? 
En contraposición, en la tercera parte se plantea un análisis a realizar de manera conjunta, dirigido por el docente, con el que ilustrar una forma distinta de abordar uno de los problemas que constituyeron la segunda parte. Con esta última sección lo que se pretende es hacer consciente al estudiante de que no existe un único camino hacia la resolución de un ejercicio, sino que puede llegarse a ella mediante distintas estrategias, siempre que los razonamientos y el conocimiento químico subyacente sean correctos.

\section{Actividades del Texto 3:}

a. Análisis de la metodología que emplea Holmes para resolver el problema y la forma en que realiza los cálculos².

b. Holmes y Watson discuten sobre las unidades de medida porque Sherlock emplea para los cálculos el sistema francés y no el inglés.

$<<$ "Holmes, why do you convert our British units to those used by the French?"

"Watson, the winds of change from the French Revolution have blown new ideas in many directions including the system of metric units. With no disloyalty to my English roots, I must say that the metric system is far superior to our English system for scientific calculations." >>

(Waddell and Rybolt, 1993)

i. Reflexiona sobre los problemas que podrían producirse si cada país tuviera un sistema de unidades propio.

ii. ¿Cómo ha solucionado la comunidad científica esos problemas?

1. ¿Te parece una buena idea?

iii. Explica cómo tenemos que trabajar los científicos, en qué sistema, indicando sus magnitudes y unidades fundamentales

1. ¿Cuáles son las ventajas de esta forma de trabajo?

c. Holmes explica a Watson que hay un nuevo concepto que se está volviendo muy popular, el concepto de mol. ¿Sabrías dar una definición de este concepto?

$<$ " I should mention to you that a term which has not yet come to general use, but which I find most useful is the concept of a mole, >>

(Waddell and Rybolt, 1993)

d. En el texto se mencionan las leyes de Boyle, Charles, Avogadro y de los Gases ideales

$\ll$ A combination of the laws of Boyle, Charles, and Avogadro will provide the final clue. Consider the perfect gas law where pressure times volume is proportional to moles times temperature. >>

\footnotetext{
${ }^{2}$ Se pretende que este análisis se haga conjuntamente, estando guiado por el profesor, ya que puede ser una actividad interesante mostrar a los estudiantes que no hay una única forma de afrontar los problemas, sino que, mientras los razonamientos sean correctos existen multitud de estrategias posibles.
} 
(Waddell and Rybolt, 1993)

i. Explica en qué consiste cada una de ellas, aportando las expresiones matemáticas oportunas cuando sea preciso. Explica en qué consiste cada una de ellas, aportando las expresiones matemáticas oportunas cuando sea preciso.

Adicionalmente, y como complemento a la actividad, puede plantearse una experiencia de cátedra que sirva como elemento de ampliación, en la que se ilustre la reacción entorno a la cual gira todo el misterio del texto. Con esta práctica puede darse entrada a una gran variedad de cuestiones con las que trabajar contenidos muy distintos a los que se aplicaron en las partes anteriores, ofreciendo una experiencia educativa mucho más completa con la que, además, pueda demostrarse que los conocimientos no forman compartimentos estancos, sino que todo está relacionado.

\section{Experiencia de cátedra:}

El objetivo principal que se persigue es que los estudiantes puedan observar una reacción química en la que intervienen sustancias en los tres estados de agregación de la materia y entorno a la cual gira el texto, trabajando los conceptos de estequiometría asociados a ella. Del mismo modo se pretende hacer consciente al alumnado de que vivimos rodeados de sustancias químicas, y que se pueden llevar a cabo reacciones muy sencillas, pero muy impresionantes con materiales de los que se dispone en cualquier casa. Para llevar a cabo la reacción lo único que necesitamos es:

- Una botella de agua de $50 \mathrm{cL}$

- Un globo

- Vinagre

- Bicarbonato sódico

En primer lugar, se vierte una cierta cantidad de vinagre en la botella, hasta un volumen aproximado de $1 / 4$ de la misma. Después, se introduce una cucharada grande de bicarbonato sódico en el globo, y se introduce el cuello de la botella en el mismo, por la parte donde se sopla para hincharlo, sin dejar caer el bicarbonato sobre el vinagre. Finalmente, se vuelca el contenido de globo, dejando que caiga a la botella y se observa lo que ocurre.

Inmediatamente comienza a producirse la reacción entre el vinagre y el bicarbonato, y a generase una espuma que comienza a subir por la botella, y un gas que comienza a hinchar el globo. Resulta bastante interesante para los educandos, tras dejar que se produzca la reacción, emplear el gas recogido en el globo para apagar una vela.

La experiencia, se basa en la reacción ácido base que se produce entre el ácido acético, un ácido débil que se encuentra en vinagre, y el ion bicarbonato, que es anfótero y puede actuar como base débil. En dicha reacción se genera $\mathrm{CO}_{2}$ que es el gas que provoca el burbujeo que se puede observar y que posteriormente llena el globo hinchándolo.

Tras llevar a cabo la experiencia se puede plantear una pequeña cuestión con la que trabajar un poco los contenidos relacionados con la estequiometría:

a. Los hermanos Joseph y Étienne Montgolfier tenían un sueño, querían volar como los pájaros, y para lograrlo, tras mucho pensar, inventaron el famoso globo aerostático. Uno de los problemas de estos pioneros era cómo hinchar su globo, porque éstos suelen tener un volumen aproximado de unos $2250 \mathrm{~m}^{3}$. Suponiendo 
que el día 21 de noviembre de 1783 en París la temperatura era de 25드 y la presión de $1 \mathrm{~atm}$, y que estos hermanos, tenían todo el vinagre que quisieran a su disposición:

i. ¿Podrías echarles una mano indicándoles cuanto bicarbonato sódico necesitarían para hinchar su fascinante invento?

ii. ¿Cuánta agua y cuánto acetato sódico se formarían?

iii. ¿Crees que esta reacción podría emplearse para hacer volar los globos? ¿Por qué o por qué no? Una pequeña pista, mira detenidamente los datos que se ofrecen al final del problema.

Datos adicionales: $\mathrm{Mw}(\mathrm{Na})=23 \mathrm{~g} \mathrm{~mol}^{-1} ; \mathrm{Mw}(\mathrm{H})=1 \mathrm{~g} \mathrm{~mol}^{-1} ; \mathrm{Mw}(\mathrm{C})=12 \mathrm{~g} \mathrm{~mol}^{-1} ; \mathrm{Mw}(\mathrm{O})=16 \mathrm{~g}$ $\mathrm{mol}^{-1}$ densidad del aire $=1 \mathrm{~g} \mathrm{ml}^{-1} \mathrm{y}$ densidad del $\mathrm{CO}_{2}=1,5 \mathrm{~g} \mathrm{ml}^{-1}$.

b. Investiga sobre los gases que se emplean para hacer volar los globos aerostáticos.

\section{Cuestiones complementarias sobre la experiencia:}

A partir de la experiencia y una vez hemos entrado en el fascinante mundo de los globos aerostáticos, a los que tantas referencias pueden encontrarse en la literatura juvenil, y para muestra el reiterado uso de ellos que hacía el padre de la ciencia ficción, Julio Verne; puede plantearse un ejercicio adicional, con el que trabajar los contenidos relacionados con el Principio de Arquímedes y el empuje ${ }^{3}$.

a. Determina, aplicando el Principio de Arquímedes, la carga máxima que podría levantar el globo de los hermanos Montgolfier, cuyo volumen era de $2250 \mathrm{~m}^{3}$, si lo hinchásemos con $\mathrm{H}_{2}$. Ten en cuenta que la masa del globo es la masa de la caja y los pasajeros más la masa del gas que llena el globo, y considera que el volumen de aire desalojado es prácticamente equivalente al volumen de gas que se necesita para llenar el globo. Datos: $\rho($ aire $)=1,225 \mathrm{~kg} / \mathrm{m}^{3} \rho\left(\mathrm{H}_{2}\right)=1,225 \mathrm{~kg} / \mathrm{m}^{3}$.

i. Comprueba lo que ocurriría si en lugar de $\mathrm{H}_{2}$ empleásemos $\mathrm{CO}_{2}$ para llenar el globo $\rho\left(\mathrm{CO}_{2}\right)=1,500 \mathrm{~kg} / \mathrm{m}^{3}$

b. Investiga un poco sobre el $\mathrm{H}_{2}$, sus características, sus propiedades, para qué se usa, cómo se produce....etc.

c. Explica por qué se usan gases como hidrógeno y helio, que son mucho más caros y difíciles de producir que otros en los globos aerostáticos.

i. ¿Por qué crees que ahora se emplea helio en los dirigibles en lugar de hidrógeno?

\footnotetext{
${ }^{3}$ Problema adaptado de (Last, 2012). Puede abordarse este mismo ejercicio, u otros similares, empleando la película "Up" como recurso, tal como se explica en (Pereyra, 2013)
} 


\section{Referencias}

Avalos, SH (2006). Experimentos de química recreativa con sulfato de cobre pentahidratado. Revista Eureka sobre Enseñanza y Divulgación de las Ciencias, 3(3), 467-484.

Jacobsen, EK (2011). Sherlock Holmes Goes Virtual. Journal of Chemical Education, 88(4), 368- 369. https://doi.org/10.1021/ed200021z

Last, AM (2012). Chemistry in Victorian Detective Fiction: "A Race with the Sun". Journal of Chemical Education, 89(5), 636-639. https://doi.org/10.1021/ed200110z

Martín, J y Vicente, MA (2019). Narrativa como recurso didáctico en Física y Química. En C López Esteban, Aquilafuente: Vol. 276. De la innovación a la investigaciión en las aulas. Modelos y Experiencias en el Máster en Profesor de Educación Secundaria Obligatoria y Bachillerato, Formación Profesional y Enseñanzas de Idiomas (1a, pp. 295-334). Salamanca: Ediciones Universidad Salamanca.

Pereyra, J (2013, mayo 13). Respuestas IX: Levantar una casa con globos (Up). Recuperado 28 de mayo de 2018, de Ciencia de Sofá, website: http://cienciadesofa.com/2013/05/respuestas-ix-up.html

Pérez, JPL y Gil, RB (2012). Una reacción química de cine. Revista Eureka sobre Enseñanza y Divulgación de las Ciencias, 9(2), 274277.

Rybolt, TR, \& Waddell, TG (1995). The Chemical Adventures of Sherlock Holmes: The Problem of Woolthshrap Prison. Journal of $\begin{array}{lll}\text { Chemical } & \text { Education, } & \end{array}$ https://doi.org/10.1021/ed072p1090

Rybolt, TR, \& Waddell, TG (1999). The Chemical Adventures of Sherlock Holmes: The Death Puzzle at 221B Baker Street. Journal of Chemical Education, 76(4), 489. https://doi.org/10.1021/ed076p489

Rybolt, TR, \& Waddell, TG (2002). The Chemical Adventures of Sherlock Holmes: The Case of Three. Journal of Chemical Education, 79(4), 448. https://doi.org/10.1021/ed079p448

Sarria, MLP, Corominas, J y Cañón, GP (2014). Química de los medicamentos de hierro: Propuestas educativas contextualizadas. Anales de la Real Sociedad Española de Química, (3), 218-224.

Shaw, K (2008). The Chemical Adventures of Sherlock Holmes: The Serpentine Remains. Journal of Chemical Education, 85(4), 507. https://doi.org/10.1021/ed085p507

Shaw, K (2009). The Chemical Adventures of Sherlock Holmes: Mrs. Hudson's Golden Brooch. Journal of Chemical Education, 86(4), 443. https://doi.org/10.1021/ed086p443
Shaw, K (2012). The Chemical Adventures of Sherlock Holmes: The Brief Case of the Vile Humour. Journal of Chemical Education, 89(5), 640-642. https://doi.org/10.1021/ed101127g

Waddell, TG, \& Rybolt, TR (1989). Sherlock Holmes and the yellow prisms. Journal of Chemical Education, 66(12), 981. https://doi.org/10.1021/ed066p981

Waddell, TG, \& Rybolt, TR (1990). Sherlock Holmes and the fraudulent ketone. Journal of Chemical Education, 67(12), 1006. https://doi.org/10.1021/ed067p1006

Waddell, TG, \& Rybolt, TR (1991). The chemical adventures of Sherlock Holmes: A Christmas story. Journal of Chemical Education, 68(12), 1023. https://doi.org/10.1021/ed068p1023

Waddell, TG, \& Rybolt, TR (1992). The chemical adventures of Sherlock Holmes: The case of the screaming stepfather. Journal of $\begin{array}{lll}\text { Chemical } & \text { Education, } & \text { 69(12), }\end{array}$ https://doi.org/10.1021/ed069p999

Waddell, TG, \& Rybolt, TR (1993). The chemical adventures of Sherlock Holmes: The case of the stoichiometric solution. Journal of Chemical Education, 70(12), 1003. https://doi.org/10.1021/ed070p1003

Waddell, TG, \& Rybolt, TR (1994). The Chemical Adventures of Sherlock Holmes: The Hound of Henry Armitage. Journal of Chemical Education, 71(12), 1049. https://doi.org/10.1021/ed071p1049

Waddell, TG, \& Rybolt, TR (1996). Sherlock Holmes and the Nebulous Nitro. Journal of Chemical Education, 73(12), 1157. https://doi.org/10.1021/ed073p1157

Waddell, TG, \& Rybolt, TR (1998). The Chemical Adventures of Sherlock Holmes: The Baker Street Burning. Journal of Chemical Education, 75(4), 484. https://doi.org/10.1021/ed075p484

Waddell, TG, \& Rybolt, TR (2000). The Chemical Adventures of Sherlock Holmes: The Ghost of Gordon Square. Journal of Chemical Education, 77(4), 471. https://doi.org/10.1021/ed077p471

Waddell, TG, \& Rybolt, TR (2001). The Chemical Adventures of Sherlock Holmes: The Shroud of Spartacus. Journal of Chemical Education, 78(4), 470. https://doi.org/10.1021/ed078p470

Waddell, TG, \& Rybolt, TR (2003). The Chemical Adventures of Sherlock Holmes: The Blackwater Escape. Journal of Chemical Education, 80(4), 401. https://doi.org/10.1021/ed080p401

Waddell, TG, \& Rybolt, TR (2004). The Chemical Adventures of Sherlock Holmes: Autopsy in Blue. Journal of Chemical Education, 81(4), 497. https://doi.org/10.1021/ed081p497 


\section{Anexo I: Obtención de cobre por cementación}

\section{Objetivos:}

1. Obtención de cobre metálico a partir de una disolución de cobre (II) por el método de cementación.

2. Comprobación experimental de la reacción que se explica en la lectura

\section{Fundamento teórico:}

Este método se basa en la reducción del catión divalente Cu2+ a cobre metálico, Cu0, por medio de una reacción de transferencia de electrones espontánea, en la que, para reducirse, toma dos electrones procedentes de la oxidación del hierro metálico, Fe0, al catión divalente $\mathrm{Fe}^{2+}$.

La espontaneidad de una reacción redox viene dada por la variación de la energía libre de Gibbs, $\Delta \mathrm{G}^{\circ}$, así, cuándo esa energía es menor que cero, decimos que la reacción es espontánea. Debemos señalar que el criterio de espontaneidad de una reacción no determina su velocidad, la cual dependerá exclusivamente de factores cinéticos y no de factores termodinámicos.

\section{Datos y fórmulas:}

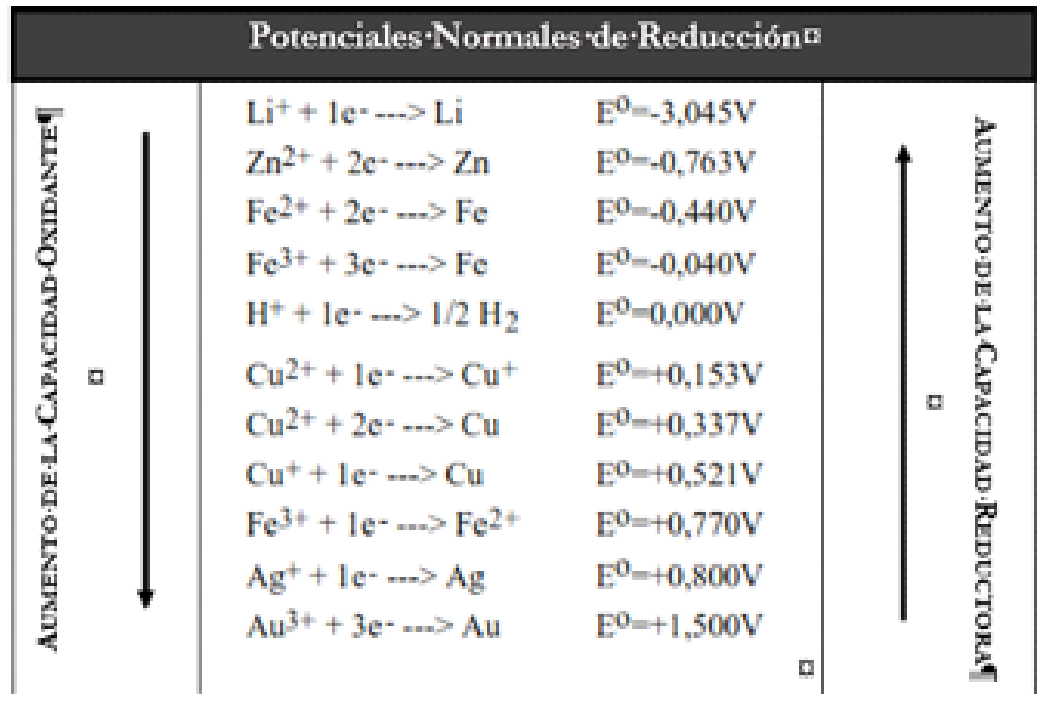

Figura 1: Potenciales estándar de reducción.

$$
\Delta G^{o}=-n \times F \times E_{r}^{o}
$$

$\mathrm{n}=$ número de electrones intercambiados

$\mathrm{F}=$ constante de Faraday $=96500 \mathrm{C} / \mathrm{mol}$

$E_{r}^{0}=$ potencial normal de la reacción

Recuerda que $\mathrm{J}$ [Julios] $=\mathrm{C}$ [Culombios $] \times \mathrm{V}$ [Voltios] 


\section{Sección experimental:}

$\underline{\text { Reactivos: }}$ sulfato de cobre (II) pentahidratado; ácido sulfúrico, agua destilada, clavos de hierro.

Materiales: balanza, vasos de precipitados, placa calefactora, sistema de filtrado a presión reducida,

\section{Procedimiento:}

En un vaso de precipitados se disuelven $5,0 \mathrm{~g}$ de sulfato de cobre(II) pentahidratado, en $50 \mathrm{~cm} 3$ de agua destilada a la que se han añadido $2 \mathrm{~mL}$ de ácido sulfúrico concentrado. Se calienta moderadamente la disolución $\left(\approx 70^{\circ} \mathrm{C}\right)$ y se añaden de cuatro a siete puntas de hierro. Si se añaden los clavos antes de calentar la deposición del cobre metálico se ralentiza considerablemente o incluso se bloquea. Se deja que progrese la reacción hasta que se observe la desaparición del color azul del Cu2+ y su sustitución por un color verde pálido indicativo de la presencia de Fe2+ en la disolución.

Se deja enfriar, se decanta y se filtra, lavando varias veces en la placa filtrante con agua para eliminar los restos de ácido sulfúrico y de cationes que impregnan a la masa de cobre. Se quita el vacío y se lava el sólido depositado en la placa una última vez con una pequeña porción de etanol. Se conecta nuevamente el sistema de vacío para que la succión y el paso de aire sequen el precipitado. Con la ayuda de un imán se retiran los restos de hierro metálico que no haya reaccionado, se completa el secado del cobre en estufa $\left(\approx 60^{\circ} \mathrm{C}, 5\right.$ minutos) y se pesa para determinar la cantidad de cobre obtenido.

\section{Cuestiones complementarias 4 :}

1. Determina el rendimiento del proceso.

2. Qué funciones tiene el ácido sulfúrico?

3. ¿Por qué se ha de calentar la disolución casi a ebullición?

4. ¿Por qué se lava el cobre con etanol?

5. Justifica termodinámicamente, que la reacción propuesta por Holmes es más favorable que el resto de las que podrían producirse como, por ejemplo:

$$
\begin{gathered}
3 \mathrm{Cu}^{2+}+2 \mathrm{Fe} \rightarrow 3 \mathrm{Cu}+2 \mathrm{Fe}^{3+} \\
2 \mathrm{Cu}^{2+}+\mathrm{Fe} \rightarrow 2 \mathrm{Cu}^{+}+2 \mathrm{Fe}
\end{gathered}
$$

6. Si la cucharilla que introdujo Sherlock en el tarro de las grosellas hubiese sido de plata, en vez de acero, ¿Se hubiera recubierto de cobre? Justifica tu respuesta sabiendo que la reacción que puede producirse es:

$$
2 \mathrm{Ag}++\mathrm{Cu} \rightarrow 2 \mathrm{Ag}+\mathrm{Cu}^{2+}
$$

\footnotetext{
${ }^{4}$ El guion puede ser completado con alguna de las ideas que se proponen en (Avalos, 2006), donde se recogen una serie de experiencias de química recreativa que tienen como protagonista al sulfato de cobre pentahidratado, principal reactivo de la práctica.
} 
7. Cuando ya no quedan iones $\mathrm{Cu} 2+$ en disolución se observa cómo empiezan a desprenderse burbujas de $\mathrm{H} 2$, de donde se deduce que ha de haberse producido la reducción de los protones de acuerdo a la ecuación:

$$
2 \mathrm{H}^{+}+2 \mathrm{e}^{-} \rightarrow \mathrm{H}_{2}
$$

(a) ¿Qué especie habrá actuado como reductor, el Fe o el Cu? Las reacciones que podrían producirse serían las siguientes:

$$
\begin{aligned}
& \mathrm{Fe}+2 \mathrm{H}^{+} \rightarrow \mathrm{H}_{2}+\mathrm{Fe}^{2+} \\
& \mathrm{Cu}+2 \mathrm{H}^{+} \rightarrow \mathrm{H}_{2}+\mathrm{Cu}^{2+} .
\end{aligned}
$$




\section{Anexo II: Obtención de cobre por cementación.}

\section{Objetivos:}

1- Observación de diferentes reacciones de formación de complejos.

Fundamento teórico:

Llamamos complejo, o compuesto de coordinación, a las especies químicas formadas por un átomo o ion metálico central, rodeado por una serie de átomos, iones o moléculas que denominamos ligandos. La reacción de formación de este tipo de compuestos puede entenderse como una reacción entre un ácido y una base de Lewis.

El átomo de un ligando que se une al átomo central se denomina donador, y el número de donadores que rodean al ion central constituye el número de coordinación. Los iones complejos pueden ser catiónicos, anicónicos o neutros, según la carga resultante de la especie.

Las disoluciones de tiocianato de amonio, $\mathrm{NH}_{4}(\mathrm{SCN})$, son incoloras, sin embargo, al reaccionar con el catión $\mathrm{Fe}^{3+}$ se genera el catión complejo tiocianato de hierro (III), $[\mathrm{Fe}(\mathrm{SCN})]^{2+}$, que tiene un característico color rojo sangre. Se trata de una reacción de equilibrio cuya ecuación viene dada por la expresión:

$$
\mathrm{Fe}^{3+}+\mathrm{SCN}-\leftrightarrow[\mathrm{Fe}(\mathrm{SCN})]^{2+}
$$

En la segunda reacción, catión $\mathrm{Cu}^{2+}$, en disolución acuosa forma un catión complejo, $\left[\mathrm{Cu}\left(\mathrm{H}_{2} \mathrm{O}\right)_{4}\right]^{2+}$ de color azul claro, cuyas dos cargas positivas son compensadas por las dos cargas negativas del anión sulfato, $\mathrm{SO}_{4}{ }^{2}$ - procedente del sulfato de cobre pentahidratado. A medida que añadimos hidróxido de amonio, $\mathrm{NH}_{4} \mathrm{OH}$, este reacciona con el complejo y da lugar a la formación de un precipitado de color azulado. Si seguimos añadiendo hidróxido de amonio, este exceso redisuelve el precipitado y da lugar a la formación del catión complejo $\left[\mathrm{Cu}\left(\mathrm{NH}_{3}\right)_{4}\right]^{2+}$, de color azul oscuro.

Reacción 2: $\left[\mathrm{Cu}\left(\mathrm{H}_{2} \mathrm{O}\right)_{4}\right] \mathrm{SO}_{4}+2 \mathrm{NH}_{4} \mathrm{OH} \rightarrow$ Precipitado $+\left(\mathrm{NH}_{4}\right)_{2} \mathrm{SO}_{4}+4 \mathrm{H}_{2} \mathrm{O}$

$$
\begin{gathered}
\mathrm{NH}_{3}(\mathrm{aq}) \leftrightarrow \mathrm{NH}_{3}+\mathrm{H}_{2} \mathrm{O} \\
\text { Precipitado }+4 \mathrm{NH}_{3} \rightarrow\left[\mathrm{Cu}\left(\mathrm{H}_{2} \mathrm{O}\right)_{4}\right]^{2+}+2 \mathrm{OH}^{-}+4 \mathrm{H}_{2} \mathrm{O}
\end{gathered}
$$

\begin{tabular}{|c|c|c|}
\hline & Parte 1 & Parte 2 \\
\hline Reactivos & $\begin{array}{c}\text { Disolución } 0.5 \mathrm{M} \text { de cloruro de hierro (III) } \\
\text { Disolución } 0.5 \mathrm{M} \text { de tiocianato de amonio } \\
\text { Agua del grifo }\end{array}$ & $\begin{array}{c}\text { Sulfato de cobre (II) pentahidratado. } \\
\text { Amoníaco }(25 \% \mathrm{p} / \mathrm{p}) \\
\text { Agua destilada }\end{array}$ \\
\hline Materiales & $\begin{array}{l}\text { Pipeta Pasteur de vidrio } \\
\text { Bandeja de disección }\end{array}$ & $\begin{array}{c}\text { Balanza } \\
\text { Vaso de precipitados } \\
\text { Probeta } \\
\text { Varilla de vidrio }\end{array}$ \\
\hline
\end{tabular}

\section{Sección experimental:}

Tabla 3: Reactivos y materiales. 


\section{Procedimiento:}

- Parte 1: se reproduce el procedimiento indicado en (Pérez y Gil, 2012).

Sobre la bandeja de disección se verterán 1 litro de agua del grifo y $20 \mathrm{ml}$ de la disolución de tiocianato de amonio. A continuación, y mediante una tetina acoplada a la pipeta Pasteur, se llenará ésta con la disolución de la sal de hierro. Cuando la pipeta se introduce en la bandeja de disección, la reacción química observable es sorprendente. Un color rojo sangre empezará a formarse en las inmediaciones de la pipeta que, tras remover el agua de la bandeja, hará que toda vire hacia este color. De manera complementaria, se puede hacer un experimento adicional eliminando el compuesto de amonio del agua, comprobándose que no sucede lo mismo que cuando estaba presente.

\section{- Parte 2:}

En un vaso de precipitados se disuelven $5,0 \mathrm{~g}$ de sulfato de cobre pentahidratado en unos $15 \mathrm{~mL}$ de agua destilada. En una probeta preparan $35 \mathrm{~mL}$ de amoniaco diluido $(25 \% \mathrm{p} / \mathrm{p})$. A continuación, se añade una pequeña porción de hidróxido de amonio mientras se agita con una varilla de vidrio. En este momento debería comenzar a apreciarse la formación de un precipitado azulado. Se continúa añadiendo hidróxido amónico poco a poco, en pequeñas porciones, hasta que la disolución adquiere un color azul oscuro y se ha gastado todo el hidróxido amónico preparado.

\section{Cuestiones complementarias ${ }^{5}$ :}

1) Los complejos son un ejemplo de ácidos y bases de Lewis, describe, de acuerdo a esta teoría lo que es un ácido y una base.

a) Recuerda otras teorías ácido base que conozcas y explica cómo se definen las sustancias ácidas y básicas en ellas indicando algún ejemplo.

2) La formación del tiocianato de hierro (III) es una reacción de equilibrio.

a) Explica en qué consiste este tipo de reacciones.

b) Enuncia el principio de Principio de Le Châtelier, indica qué factores son los que afectan al desplazamiento del equilibrio y cómo lo hacen, aportando una serie de ejemplos.

c) ¿Qué ocurriría si añadiésemos $\mathrm{Fe}^{3+}$ a la disolución una vez se haya alcanzado el equilibrio? ¿Y si añadiéramos tiocianato de hierro (III)?

3) En la Reacción 2 se indica que se ha formado un precipitado.

a) ¿Podrías decir cuál es el precipitado que se ha formado?

b) Si quisiéramos obtener una mayor cantidad de dicho precipitado, pero no dispusiéramos de más cantidad de cobre ¿Qué deberíamos hacer?

\footnotetext{
${ }^{5}$ Sarria, Corominas, y Cañón, (2014) recogen una serie de experiencias y ejercicios que tienen al hierro como protagonista y pueden ser un magnífico complemento para completar la experiencia del alumnado, así como para aportar un contexto cotidiano, el de la medicina, a algunos de los contenidos que se pretenden trabajar con esta práctica de laboratorio.
} 


\section{Anexo III: Textos para la actividad sobre The Case of the Stoichiometric Solution.}

Los tres textos seleccionados pertenecen a la contribución original de los autores (Waddell \& Rybolt, 1993), publicada en el Journal of Chemical Education y no serán reproducidos aquí. No obstante, se indica a qué parte de la publicación original corresponde cada una de las selecciones que se han hecho para el desarrollo de la actividad.

\section{Texto 1: Un Nuevo Caso para Sherlock Holmes}

Abarca las páginas 1003 y 1004 del artículo, en concreto, desde el comienzo hasta el párrafo:

"It all fits, Watson. The pock-marked, sour-smelling hole we found was formed when the murderer dropped some acetic acid on the limestone. Remember from your school days; we could write the equation as," said Holmes as he scratched upon a piece of paper.

(Waddell \& Rybolt, 1993) páginas 1003-1004

\section{Texto 2: Desentrañando el misterio:}

Comienza justo a continuación de donde terminó el anterior, en la página 1004, en concreto en el párrafo:

"But, Holmes, where is the poison?"

Finaliza en la misma página, concretamente en el fragmento siguiente:

"Stoichiometry, my dear fellow. Surely you recall from your school days the utility of the definite relations of mass, volume, and amount between constituents in chemical reactions."

(Waddell \& Rybolt, 1993) página 1004

\section{Texto 3: Los Cálculos de un Detective:}

Se inicia en la página 1004, y se extiende hata el final de la publicación, comenzando en el párrafo siguiente:

"Well, I am not certain I recall all the details which I may have learned. I am afraid I still don't see how this provides the key."

(Waddell \& Rybolt, 1993) páginas 1004-1005 